\title{
Uma associação do método Peer Instruction com circuitos elétricos em contextos de aprendizagem ativa
}

\section{An association of Peer Instruction method with electrical circuits in active learning contexts}

\author{
A. V. R. de Araujo*1, E. S. Silva ${ }^{2}$, V. L. B. de Jesus ${ }^{3}$, A. L. de Oliveira ${ }^{3}$ \\ ${ }^{1}$ Instituto Federal de Educação, Ciência e Tecnologia do Rio de Janeiro, campus Pinheiral, RJ, Brasil \\ ${ }^{2}$ Instituto Federal de Educação, Ciência e Tecnologia de Mato Grosso, campus Juína, MT, Brasil \\ ${ }^{3}$ Instituto Federal de Educação, Ciência e Tecnologia do Rio de Janeiro, Nilópolis, RJ, Brasil
}

Recebido em 19 de Agosto, 2016. Revisado em 06 de Outubro, 2016. Aceito em 08 de Outubro, 2016.

Neste trabalho são discutidos os resultados de uma sequência didática baseada no método Peer Instruction para o ensino de circuitos elétricos, aplicada em sete turmas de ensino médio integrado a cursos técnicos. Os dados obtidos, em dois testes iguais, antes e depois da aplicação do método, foram analisados a partir do ganho normalizado ou ganho de Hake, e comparados a trabalhos na literatura nacional e internacional. Os resultados revelam que seis delas apresentaram ganhos compatíveis com aqueles esperados para turmas submetidas a estratégias de aprendizagem ativa, de acordo com a literatura nacional. Por outro lado, somente os resultados apresentados por três turmas permitiriam classificá-las como turmas em que ocorreu aprendizagem ativa, considerando valores estabelecidos na literatura internacional. Nossos resultados corroboram a ideia de que a sequência didática pode ser utilizada como mediadora da relação ensino-aprendizagem para a discussão de circuitos elétricos.

Palavras-chave: Peer Instruction, circuitos elétricos, Plickers, ganho de Hake, aprendizagem ativa.

In this paper the results of a didactic sequence based on the Peer Instruction method for the teaching of electrical circuits are discussed. The didactic sequence was applied in seven classes of integrated technical courses at high school level. The data obtained in two equal tests before and after the application of the method were analyzed from the normalized gain or gain of Hake, and compared with Brazilian and international literature. The results show that six of them had consistent normalized gains with those expected for classes under active learning strategies, according to the Brazilian literature. On the other hand, only the results reported by three groups allow classifying them as classes where there was active learning, considering values established in the international literature. Our results strength the idea that the teaching sequence can be used as a mediator of the teaching-learning for the discussion of electrical circuits.

Keywords: Peer Instruction, electric circuits, Plickers, normalized gain, active learning.

\section{Introdução}

Encontramos, com frequência, cursos de física baseados em aulas expositivas e na resolução de exercícios padronizados. Por diversas razões - tempo, infraestrutura e deficiências na formação - seus professores deixam de lado a realização de experimentos. Apesar de possuir caráter experimental, a física, nas escolas, ainda é ministrada com enfoque fortemente teórico [1].

*Endereço de correspondência: alexandre.araujo@ifrj.edu.br
Trabalhos publicados nas últimas décadas apresentaram aplicações de metodologias alternativas, capazes de modificar a estrutura de uma aula tradicional de física $[2,3]$. As estratégias de aprendizagem ativa tem se destacado na literatura internacional, sendo apresentadas como opções viáveis para implantação no ensino médio. Elas permitem maior engajamento dos alunos, utilizando um estudo prévio, observação de fenômenos físicos e interpretação de testes conceituais [4]. 
Um dos métodos de aprendizagem ativa, que existe desde o início dos anos 1990, é denominado Peer Instruction (PI) [4]. Em publicações brasileiras, essa metodologia é conhecida como Instrução por Pares [3] ou Instrução pelos Colegas - IpC [5]. Sua aplicação permite que os alunos assumam papéis mais ativos durante as aulas, em momentos de discussões com os colegas, quando estão resolvendo testes conceituais referentes aos tópicos em estudo. Durante essa troca de ideias, o professor atua como um mediador, orientando seus alunos e incentivando o debate entre eles.

Algumas dissertações de mestrado brasileiras apontam estratégias para adoção da Peer Instruction, associadas com outras metodologias ativas e adaptadas para a nossa realidade. Os trabalhos de Diniz [6] e Müller [7] apresentam pesquisas realizadas em turmas de ensino médio, em dois Colégios de Aplicação. Oliveira [8] também pesquisou o método, aplicando-o em uma turma de terceira série do Instituto Federal de Educação, Ciência e Tecnologia Sul-rio-grandense (IFSul), campus Pelotas.

Artigos nacionais sobre o tema, em contextos de ensino médio, ainda são escassos e surgem, principalmente, como desdobramentos de pesquisas de mestrado. Por exemplo, Müller [9] e Oliveira [10] publicaram trabalhos relacionados com suas dissertações, ambos no Caderno Brasileiro de Ensino de Física.

No presente trabalho, utilizamos a Peer Instruction, em conjunto com demonstrações de experimentos em circuitos elétricos, em turmas de ensino médio integrado a cursos técnicos de agropecuária, meio ambiente e informática, no Instituto Federal de Educação, Ciência e Tecnologia do Rio de Janeiro, campus Pinheiral. Os questionamentos e atividades foram estruturados com o apoio de circuitos elétricos simples, formados por uma fonte de tensão elétrica e lâmpadas incandescentes. A metodologia adotada e os resultados obtidos serão detalhados nas próximas seções.

\section{O método Peer Instruction}

Peer Instruction é um método de aprendizagem ativa que possui dois objetivos básicos: explorar a interação entre os estudantes e focar sua atenção nos conceitos fundamentais para a resolução de questionamentos propostos em sala. Nesta metodologia, os alunos possuem maior autonomia para discussões em sala de aula, a respeito de testes conceituais propostos pelo professor. A técnica surgiu a partir da prática docente de seu idealizador, o professor de física Eric Mazur, na Universidade de Harvard. Ele recomenda que os alunos realizem a leitura prévia indicada, extraclasse, de um determinado assunto a ser discutido. Durante a aula, onde será trabalhado o assunto da leitura realizada pelos alunos, podem ocorrer os seguintes passos [4]:

1. O professor realiza uma exposição oral, de aproximadamente 15 minutos, sobre os elementos mais importantes do tópico a ser trabalhado.

2. É proposto um teste conceitual, de múltipla escolha, a respeito do tema apresentado na exposição oral. Os alunos refletem sobre o teste conceitual, individualmente, de maneira silenciosa, durante $1 \mathrm{a}$ 2 minutos.

3. Cada estudante decide qual é a opção correta e registra sua resposta, mostrando-a ao professor, que fará a distribuição de acertos da turma. Quando menos de $30 \%$ da turma acerta a resposta, o professor deve repetir o passo 1 .

4. Quando entre $30 \%$ e $70 \%$ da turma escolhe a resposta correta, o professor abre espaço para discussão entre os alunos. Em duplas ou em pequenos grupos, os estudantes são encorajados a discutir suas respostas com os colegas, durante 2 a 4 minutos. Por outro lado, caso mais de $70 \%$ da turma acerte a questão, o professor explica rapidamente a resposta correta e, a seguir, propõe outro teste conceitual sobre o mesmo assunto.

5. Após a discussão, os estudantes registram novamente suas respostas, que podem ou não terem sido alteradas pela interação com os colegas, apresentandoas ao professor. Espera-se que, após as interações entre os colegas, a frequência de acertos ultrapasse $70 \%$. Desse modo, o professor pode passar para outro teste conceitual, repetindo os procedimentos enquanto houver tempo disponível de aula.

A aula é estruturada a partir dos questionamentos levantados pelos testes conceituais, conforme Mazur define. Sendo assim, a Instrução por Pares exige cuidadosa escolha dos testes trabalhados. Para auxiliar o professor nessa tarefa, existem várias fontes disponíveis, tais como vestibulares ou o Exame Nacional do Ensino Médio (ENEM). Os testes devem abordar importantes conceitos do tópico em estudo. Além disso, é recomendável que sejam desafiadores, despertando o interesse em sua resolução $[4,8]$.

A Peer Instruction já está consolidada, em outros países, geralmente associada ao ensino supe- 
rior de física, e tem sido objeto de estudo entre os pesquisadores da área [2]. Um artigo publicado na revista Physics Education Research Conference [11], apresentou resultados da aplicação do método em conteúdos de mecânica, envolvendo as leis de Newton. Os dados obtidos foram analisados a partir do ganho de Hake, em dois testes iguais, antes e depois da aplicação do método. A PI conduziu a um ganho normalizado em torno de $40 \%$, enquanto métodos tradicionais de ensino resultaram em ganho de $24 \%$. Desse modo, o artigo destacado aponta para uma contribuição da metodologia na compreensão dos conteúdos discutidos em mecânica [11]

\section{Procedimentos e técnicas utilizadas}

A pesquisa foi realizada no Instituto Federal de Educação, Ciência e Tecnologia do Rio de Janeiro (IFRJ), campus Pinheiral, em sete turmas do ensino médio regular, com um número total de 155 estudantes, de 17/05/2016 a 21/06/2016.

A carga horária da disciplina física era de dois tempos semanais, com 45 minutos de duração cada um, sem intervalo entre eles. Para fins de entendimento, adotaremos o termo aula, neste artigo, como um conjunto de dois tempos de 45 minutos cada.

Ao longo de sete aulas, foram realizadas várias atividades em cada turma, envolvendo aspectos teóricos e experimentais relacionados ao estudo de circuitos elétricos compostos por geradores e resistores. As aulas 1 e 7 foram dedicadas às aplicações do pré-teste e pós-teste. A aula 2 seguiu a estrutura convencional do método Peer Instruction, sem apresentação de experimentos. Nas demais, adotamos a sequência de procedimentos descritos a seguir:

1. Nos primeiros dez minutos de cada aula foram abordados os pontos fundamentais, teóricos, do conteúdo previsto para aquele dia.

2. Logo após a explanação teórica, apresentamos o circuito elétrico programado para a aula, identificando os componentes do circuito, o tipo de ligação entre eles e a relação entre diferença de potencial, corrente elétrica e potência elétrica, em cada uma das lâmpadas. Esses procedimentos duravam, em média, entre 5 e 10 minutos.

3. Em seguida, eram propostos testes conceituais, cujas respostas, discussões e análises de resultados seguiam o método Peer Instruction. Cada estudante recebeu uma folha impressa, com as questões que seriam resolvidas na aula. Todas as respostas foram apuradas utilizando o aplicativo Plickers, instalado no celular do professor.

4. Próximo ao final da aula era indicada uma leitura do livro texto, relativa ao assunto que seria abordado no próximo encontro.

Os testes conceituais foram selecionados de acordo com critérios estabelecidos na literatura internacional [4], ou seja, procuramos questões teóricas de múltipla escolha, que tratavam de um único assunto, com nível de dificuldade adequado à proposta da Peer Instruction

Nas aulas, foram utilizados circuitos elétricos simples, formados por uma fonte de tensão elétrica e lâmpadas incandescentes (ver Fig. 1). Ao apresentar cada circuito, comentou-se sobre a relação entre corrente elétrica e potência dissipada, em cada uma das lâmpadas. Comparando as associações em série e em paralelo, demonstrou-se que ligar ou desligar uma determinada lâmpada poderia causar efeitos em todo o circuito, não apenas no ponto onde ela estava conectada. Desse modo, ao final da sequência didática, era esperado que os alunos fossem capazes de analisar, de forma autônoma, o comportamento dos circuitos apresentados e de outros análogos.

\section{Seleção das questões de pré-teste e pós-teste}

O pré-teste pode se tornar, para o professor pesquisador, uma interessante fonte de informações a respeito das concepções prévias dos alunos. No caso dos circuitos elétricos, artigos publicados no Bra-
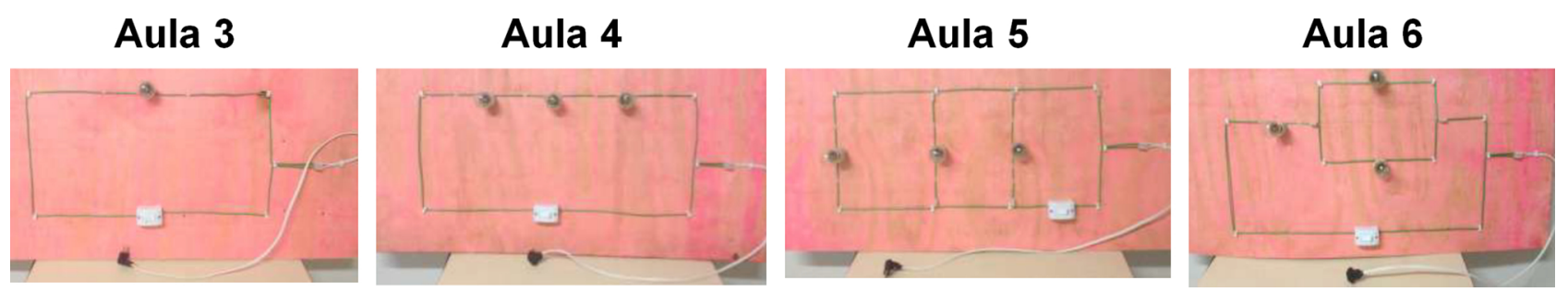

Figura 1: Circuitos apresentados em sala de aula. 
sil apresentam estudos sobre o levantamento dessas concepções. Um deles trata das ideias alternativas que os estudantes apresentam, a partir de seus conhecimentos prévios, sobre eletrodinâmica, contendo um teste que permite identificar se um estudante possui ou não concepções científicas sobre corrente elétrica em circuitos simples [12].

De acordo com Mazur [4], pré-testes e pós-testes devem ser idênticos. Em nossa pesquisa, as questões utilizadas foram adaptadas das referências [12] e [13]. A seguir, apresentamos, como exemplo, duas dessas questões:

1) (ADAPTADA) Nos circuitos I e II, a lâmpada $\mathrm{L}$, o resistor $\mathrm{R}$ e a bateria são exatamente os mesmos. Nestas situações:
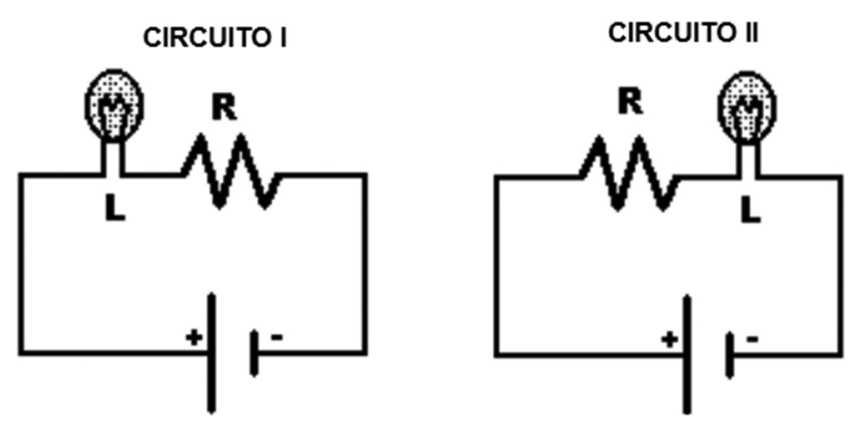

a) L brilha mais no circuito I

b) L brilha igual em ambos os circuitos.

c) L brilha mais no circuito II.

2) (ADAPTADA) Considere as quatro lâmpadas idênticas. No circuito da figura a seguir o brilho de $\mathrm{L}_{2}$ é:

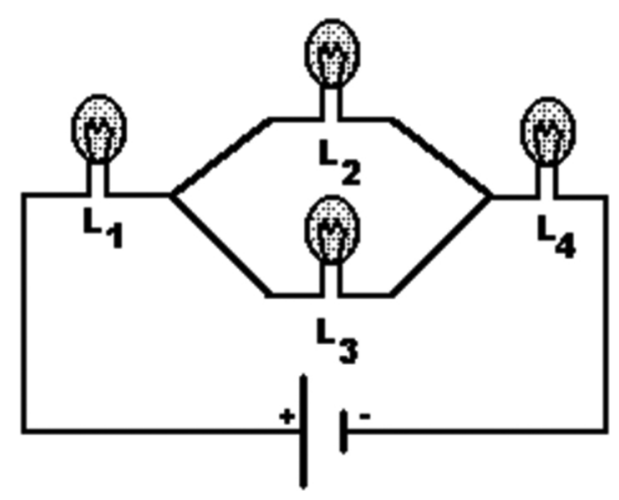

a) igual ao de $\mathrm{L}_{4}$

b) maior do que o de $\mathrm{L}_{4}$

c) menor do que o de $\mathrm{L}_{4}$
Os estudantes receberam os testes impressos e, após sua resolução, todo o material foi recolhido e posteriormente analisadd ${ }^{1}$

\section{A utilização do aplicativo Plickers}

O Plickers é um aplicativo gratuito, disponível para celulares, tablets e computadores, que permite a coleta de respostas de uma determinada turma de maneira rápida e dinâmica. É necessário criar uma conta no site [14] e cadastrar os dados da classe e questões a serem aplicadas, na biblioteca do aplicativo.

O professor pode estar interessado, por exemplo, em trabalhar 10 questões objetivas de eletrodinâmica, com uma turma de 20 alunos. Inicialmente, o docente adicionará as 10 questões na biblioteca do aplicativo, em uma pasta que pode se chamar ELETRODINÂMICA. Em seguida, ele realiza o cadastro dos 20 alunos nomeando a turma, por exemplo, TURMA-1. Os alunos podem ser identificados no aplicativo pelo nome e/ou número da chamada. Essa é a etapa relativa ao cadastro de questões e turma.

Na sala de aula, o professor pode propor uma questão aos alunos e coletar as respostas através do aplicativo. Continuando o exemplo anterior, o docente pode apurar as respostas da TURMA-1 em relação ao primeiro questionamento da pasta ELETRODINÂMICA. Os alunos leem a questão projetada em datashow ou impressa em uma folha de papel. Após escolherem a alternativa correta, votam utilizando o cartão-resposta padrão Plickers. Com uma câmera de tablet ou celular o professor realiza a leitura óptica dos cartões e o aplicativo fornece instantaneamente o percentual de acertos da turma. O cartão de votação pode ser obtido para impressão ou compra no site do Plickers

Esse aplicativo apresenta algumas vantagens, em comparação com outros recursos disponíveis. Ele armazena os dados relativos a cada votação no tablet ou celular utilizado para a leitura das respostas, não requerendo internet durante a votação. Com o Plickers, é possível apurar as respostas e obter a porcentagem de acertos em sala, sem necessidade de estar conectado à rede.

\footnotetext{
${ }^{1}$ Os materiais utilizados na pesquisa podem ser acessados no link https://drive.google.com/open?id= OBz40RYR7MgyEaEV2cDdoX2poOW8
} 
Posteriormente, quando o dispositivo utilizado se conectar à internet, o aplicativo atualiza automaticamente a conta do professor. A partir desse momento, toda a estatística pode ser acompanhada de qualquer dispositivo associado à conta do docente, pois os dados já estarão disponíveis no servidor.

\section{Resultados e discussões}

A evolução de cada turma submetida à metodologia proposta foi medida pelo parâmetro denominado ganho normalizado ou ganho de Hake [2], definida pela expressão:

$$
g=\frac{\% \text { pós }-\% \text { pré }}{100 \%-\% \text { pré }}
$$

Na Eq. 1, \%pós é a porcentagem de acertos no pós-teste, em determinada turma, e \%pré é a porcentagem de acertos no pré-teste, para a mesma turma. Os resultados dos cálculos do ganho de Hake para cada turma pesquisada estão apresentados na Tab. 1.

Hake [2] define três classes de ganho normalizado. Turmas de ganho baixo apresentam valores de $g$ $<0,30$. Nas turmas de ganho médio, os valores de $g$ estão no intervalo $0,30 \leq g<0,70$. Já as turmas com ganhos normalizados iguais ou maiores que 0,70 representam a classe de ganho alto. Nossa pesquisa aponta três turmas (MA 304, TI 305 e TI 106) na classe de ganho médio, compatíveis a cursos realizados com metodologias de aprendizagem ativa (ver Tab. 1).

Valores ligeiramente diferentes são encontrados em Mazur [4]. De acordo com esse autor, turmas Peer Instruction devem apresentar $g>0,36$. Nosso trabalho exibe apenas uma turma (TI 305) situada nessa faixa (ver Tab. 1).

Na literatura nacional, os valores encontrados são bem diferentes. Professores da Universidade Federal de Juiz de Fora (UFJF) efetuaram pesquisas envolvendo métodos de engajamento interativo, adotando o intervalo de $0,10<g<0,20$ para classes submetidas a métodos tradicionais [3]. Da Tab. 1, podemos observar que em nosso trabalho, apenas uma das turmas investigadas (TA 302) revelou ganho abaixo de 0,20 . As outras seis turmas apresentaram resultados condizentes com aqueles esperados após um processo de aprendizagem ativa.

Em trabalho mais recente, Santos [15] utilizou a Instrução por Pares em duas turmas de primeiro ano dos cursos de Ciências Exatas e Engenharia, na Universidade Federal de Itajubá. Os ganhos normalizados obtidos foram de 0,20 e 0,17 . Apenas uma de nossas turmas (TA 302) encontra-se nesta faixa.

Outra pesquisa brasileira foi desenvolvida por Diniz [6], no Colégio de Aplicação João XXIII, da UFJF. Ele aplicou a metodologia Peer Instruction em uma turma de primeira série do ensino médio, obtendo $g=0,10$. De acordo com o autor, o método não foi efetivo, ou seja, não houve um ganho significativo de aprendizagem. Todas as nossas turmas possuem valores de $g$ maiores que 0,10 .

Oliveira [10] publicou resultados de uma pesquisa realizada no Instituto Federal de Educação, Ciência e Tecnologia Sul-rio-grandense (IFSul), campus Pelotas, em uma turma de terceira série do nível médio. O autor implementou uma associação da Instrução por Pares com outro método de aprendizagem ativa, o Just in Time Teaching (JiTT).Na aplicação do $J i T T$, o professor estrutura suas aulas a partir das concepções prévias levantadas em atividades realizadas pelos alunos, em momento anterior a aula. A combinação dos métodos conduziu a $g=0,65$. Calculando um ganho médio para o nosso trabalho, bem como seu desvio padrão, obtemos $g=0,27$ $\pm 0,09$, valor inferior ao alcançado pela sequência adotada por Oliveira [10].

\section{Considerações finais}

O trabalho descrito neste artigo apresentou uma aplicação da metodologia Peer Instruction para o

Tabela 1: Dados comparativos do ganho de Hake. Número máximo de acertos: 15.

\begin{tabular}{lccccccc}
\hline Turma & TA & TA & MA & MA & TI & TI & TI \\
& 301 & 302 & 303 & 304 & 305 & 105 & 106 \\
\hline Número de alunos que fizeram o pré-teste. & 20 & 12 & 18 & 18 & 13 & 24 & 28 \\
Número de alunos que fizeram o pós-teste. & 20 & 15 & 19 & 27 & 17 & 24 & 28 \\
Porcentagem de acertos no pré-teste. & 25,3 & 29,4 & 28,9 & 31,5 & 29,7 & 33,3 & 32,6 \\
Porcentagem de acertos no pós-teste. & 41,7 & 37,8 & 47,4 & 55,3 & 55,7 & 48,1 & 55,0 \\
Ganho de Hake & 0,22 & 0,12 & 0,26 & 0,35 & 0,37 & 0,22 & 0,33 \\
\hline
\end{tabular}


ensino de circuitos elétricos, no ensino médio. Em nossa pesquisa, 3 das 7 turmas investigadas (43\%) obtiveram ganhos de Hake compatíveis com aqueles adotados pela literatura internacional, para turmas submetidas a métodos de aprendizagem ativa [2]. Levando em conta parâmetros nacionais, no que se refere ao ganho de Hake, os desempenhos de 6 das 7 turmas (86\%) são compatíveis com aqueles previstos para turmas submetidas a metodologias de aprendizagem ativa [3].

Os valores registrados na literatura internacional devem ser considerados com o devido cuidado, pois metodologias em contextos ligeiramente diferentes podem deslocar os valores de ganho normalizado, conforme aponta Barros [3]. De fato, os números apresentados por Hake [2] e Mazur [4] são relativamente altos, quando comparados aos descritos na literatura nacional. $\mathrm{O}$ trabalho que exibe resultado mais próximo da literatura internacional foi apresentado por Oliveira [10], que combinou a metodologia Peer Instruction com o JiTT, além de realizar um teste de consistência interna, a partir do coeficiente de fidedignidade alfa de Conbrach. Esse coeficiente é utilizado para estimar a confiabilidade de um determinado teste aplicado a um grupo, expressando a fração da variabilidade observada, entre indivíduos, que não pode ser atribuível a erros de medida $[8,10$, 16].

Por fim, uma investigação mais exaustiva das metodologias de aprendizagem ativa pode levar a estimativas de parâmetros mais adequados aos contextos nacionais, ajudando a delinear objetivos factíveis para o ensino de física, em nível médio, no Brasil.

\section{Agradecimentos}

Os autores agradecem ao IFRJ, campus Nilópolis, pelo suporte financeiro ao trabalho - bolsa de mestrado do Programa de Pós-graduação Stricto Sensu em Ensino de Ciências (PROPEC).

\section{Referências}

[1] H. Bonadiman e S.E.B. Nonenmacher, Caderno Brasileiro de Ensino de Física 24, 2 (2007).

[2] R.R. Hake, American Journal of Physics 66, 64 (1998).

[3] J. Acacio de Barros, Julie Remold, Glauco S.F. da Silva e J.R. Tagliati, Revista Brasileira de Ensino de Física 26, 63 (2004).
[4] E. Mazur, Peer Instruction: A Revolução da Aprendizagem Ativa (Penso, Porto Alegre 2015), $1^{\mathrm{a}}$ ed., p. 252.

[5] I. S. Araujo e E. Mazur, Caderno Brasileiro de Ensino de Física 30, 364 (2013).

[6] A.C. Diniz, Implementação do Método Peer Instruction em Aulas de Física no Ensino Médio. Dissertação de Mestrado, Universidade Federal de Viçosa, 2015.

[7] M.G. Müller, Metodologias Interativas na Formação de Professores de Fúsica: Um Estudo de Caso com o Peer Instruction. Dissertação de Mestrado, Universidade Federal do Rio Grande do Sul, 2013.

[8] V. Oliveira, Uma Proposta de Ensino de Tópicos de Eletromagnetismo Via Instrução Pelos Colegas e Ensino Sob Medida Para o Ensino Médio. Dissertação de Mestrado, Universidade Federal do Rio Grande do Sul, 2012.

[9] M.G. Müller, R.V. Brandão, I.S. Araujo e E.A. Veit, Caderno Brasileiro de Ensino de Física 29, 491 (2012).

[10] V. Oliveira, E.A. Veit e I.S. Araujo, Caderno Brasileiro de Ensino de Física 32, 180 (2015).

[11] K. Cummings and S.G. Roberts, Physics Education Research Conference 1064, 103 (2008).

[12] J.B. Rocha Filho (org.), Física no Ensino Médio: Falhas e Soluções (EDIPUCRS, Porto Alegre, 2011).

[13] O. Balen, V. Villas-Boas e F. Catelli, in: Anais do XXXVI Congresso Brasileiro de Educação em Engenharia (Associação Brasileira de Educação de Engenharia, Brasília, 2008).

[14] Www.plickers.com, acessado em 13/8/016.

[15] Z.O. Santos, A Utilização da Metodologia da Instrução pelos Colegas na Aprendizagem do Conceito de Força em Turmas das Áreas de Ciências Exatas e Engenharia. Dissertação de Mestrado, Universidade Federal de Itajubá, 2015.

[16] M.A. Moreira e F.L. da Silveira, Instrumento de Pesquisa em Ensino e Aprendizagem: A Entrevista Clínica e a Validação de Testes de Papel e Lápis (EDIPUCRS, Porto Alegre, 1993). 